2 This supplementary includes the following supporting information:

3 Supplementary Methods: Detailed description of dissolved organic carbon and dissolved oxygen flux calculations.

4 Supplementary Discussion: Discussion about the incubation of extracellular enzyme samples under $\mathrm{N}_{2}$ atmosphere.

5 Supplementary Figure 1: Extracellular enzyme rates with all samples incubated under $\mathrm{N}_{2}$ atmosphere irrespective 6 of the in situ oxygen concentration.

7 Supplementary Table 1: Cruise, date, positions, sampled depths and bottom depth of all stations represented within 8 the manuscript.

9 Supplementary Table 2: Average and standard deviation at different oxygen regimes of all discussed parameters. 


\section{Supplementary Methods:}

\section{Diapycnal fluxes of oxygen and dissolved organic carbon}

13 The mixing of DOC and oxygen across density surfaces is derived following Fischer et al. (2013) and Schafstall et 14 al. (2010). Gradients of DOC where calculated between sampled bottles assuming a constant gradient in between 15 while oxygen gradients were derived by fitting a linear trend over $3 \mathrm{~m}$ intervals, as oxygen is available on a much 16 higher vertical resolution of $1 \mathrm{dbar}$.

17 The diapycnal flux of solutes, i.e. DOC and oxygen, is calculated as

$$
\Phi_{S}=-K_{\rho} \nabla C_{S}
$$

18 Where $\nabla C_{S}$ is the vertical gradient of the solute - in case of oxygen the concretion is converted from $\mu \mathrm{mol} \mathrm{kg}^{-1}$ to

$19 \mu \mathrm{mol} \mathrm{m}{ }^{-3}$ beforehand - and $K_{\rho}$ is the diapycnal diffusivity of mass. We assume $K_{\rho}$ to be equal to the diffusivity of 20 DOC and oxygen, i.e. $K_{\rho}=K_{D O C}=K_{D O}$ as done by Fischer et al. (2013) for oxygen.

21 The diapycnal diffusivity is calculated following Osborn (1980):

$$
K_{\rho}=\Gamma \frac{\varepsilon}{N^{2}}
$$

22 Where $\Gamma=0.2$ is the mixing efficiency, $\varepsilon\left(\mathrm{m}^{2} \mathrm{~s}^{-3}\right)$ is the dissipation rate of turbulent kinetic energy calculated by 23 integrating the shear spectra derived from measurements on a freefalling microstructure probe at stations G-N \& Q-T 24 (Sea \& Sun Technology, MSS90D, S/N26 up to CTD 43, S/N 73 afterwards) (Schafstall et al., 2010), and $N\left(\mathrm{~s}^{-1}\right)$ is 25 the buoyancy frequency derived from CTD downcast profiles over 7 dbar intervals where DOC and oxygen were 26 measured as well.

27 To calculate $K_{\rho}$, data from CTD profiles we combined with nearby microstructure profiles conducted directly before 28 or after the CTD profile which existed for 12 profile. For each solutes gradient between to samples a $K_{\rho}$ value was 29 derived from dissipation and buoyancy frequency averaged between the potential densities of the two solutes 30 samples. The mean $K_{\rho}$ profile exhibits only weak vertical variations (Supplementary Figure 2a) therefore a constant $31 K_{\rho}=10^{-3} \mathrm{~m}^{2} \mathrm{~s}^{-1}$ was used to calculate fluxes.

32 For each profile a $\Phi_{S}$ profile is calculated in $20 \mathrm{~m}$ depth bins, from these a mean profile of diapycnal flux is derived.

33 Subsequent, the vertical divergence of the mean flux profile of DOC or oxygen is given by $\nabla \Phi_{S}=-\frac{\partial}{\partial z} \Phi_{S}$.

34 The error estimates of these terms are calculated following Schafstall et al. (2010), with the error of the mean $35 K_{\rho}$ given by:

$$
\Delta K_{\rho}=K_{\rho}\left[\left(\frac{\Delta \Gamma}{\Gamma}\right)^{2}+\left(\frac{\Delta \varepsilon}{\varepsilon}\right)^{2}+\left(\frac{\Delta N^{2}}{N^{2}}\right)\right]^{\frac{1}{2}}
$$


And the error of the mean flux by

$$
\Delta \Phi_{S}=\Phi_{S}\left[\left(\frac{\Delta \Gamma}{\Gamma}\right)^{2}+\left(\frac{\Delta \varepsilon}{\varepsilon}\right)^{2}+\left(\frac{\Delta N^{2}}{N^{2}}\right)+\left(\frac{\Delta \nabla C_{S}}{\nabla C_{S}}\right)\right]^{\frac{1}{2}}
$$

37 Where $K_{\rho}$ and $\Phi_{S}$ are the mean profiles calculated from the individual profiles in $20 \mathrm{~m}$ depth bins, a constant

$38 \Delta \Gamma=0.04$ is used (Schafstall et al., 2010), the error of dissipation $\Delta \varepsilon$ is the 95\%-confidence interval derived by

39 bootstrapping, $\Delta N^{2}$ and $\Delta \nabla C_{S}$ are the standard deviations of the mean. Bootstrapping and standard deviations are

40 performed on the values of the individual profiles in $20 \mathrm{~m}$ bins. The error of the change in the diapycnal flux over

41 depth is derived by error propagation from the flux.

\section{Supplementary Discussion:}

\section{Incubation of extracellular enzyme samples under $\mathbf{N}_{\mathbf{2}}$ atmosphere}

44 Enzyme rates of suboxic waters have been obtained by incubating samples under $\mathrm{N}_{2}$ atmosphere to reduce oxygen 45 concentrations. For the assessment of extracellular enzyme rates we chose to incubate samples at oxic and reduced 46 oxygen concentrations depending on oxygen concentrations at in situ depth (see methods for details). Incubation 47 conditions slightly influenced the resulting enzyme rates. Incubating samples from depths with in situ oxygen 48 concentrations $<5 \mu \mathrm{mol} \mathrm{kg}{ }^{-1}$ under $\mathrm{N}_{2}$ atmosphere, instead of under atmospheric oxygen concentrations, yielded on 49 average 2-27\% higher rates. Consequently, the differences in rates between oxic and suboxic waters became reduced, 50 when all samples were incubated under $\mathrm{N}_{2}$ atmosphere (supplementary Figure 2). However, the observed trends over 51 depth remained similar. Possible reasons for higher extracellular enzyme rates after incubation under $\mathrm{N}_{2}$ atmosphere 52 are changes in (i) $\mathrm{pH}$, (ii) the abundance of oxygen radicals and/or (iii) changes in enzyme production. (i) Higher 53 extracellular enzyme rates after incubations at low oxygen concentrations are less likely driven by the resulting 54 increase in $\mathrm{pH}(\Delta 0.4)$, since earlier studies rather suggested that extracellular enzyme rates decrease with $\mathrm{pH}$ 55 (Endres et al., 2014; Piontek et al., 2013). (ii) Oxygen radicals can destroy enzymes (Elstner, 1990). Since our 56 incubations were not completely anoxic, an influence of oxygen radicals on enzyme activity cannot be excluded, but 57 would appear under aerobic and $\mathrm{N}_{2}$ atmosphere. (iii) Finally, an enhanced production of enzymes within the 12 hours 58 of incubation time might be the reason for higher rates after incubations under $\mathrm{N}_{2}$ atmosphere. It has been shown 59 before that enzymes can be produced within 30 minutes (Both et al., 1972), making this explanation likely. The final 60 reason for higher enzyme productions under $\mathrm{N}_{2}$ atmosphere remains a matter of speculation, but supports the trends 61 seen in the depth profiles and does not restrict the interpretation of the extracellular enzyme data. 
(a)

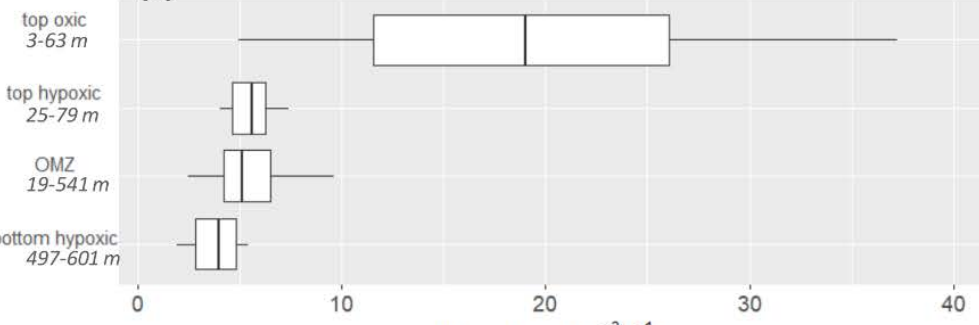

(b)

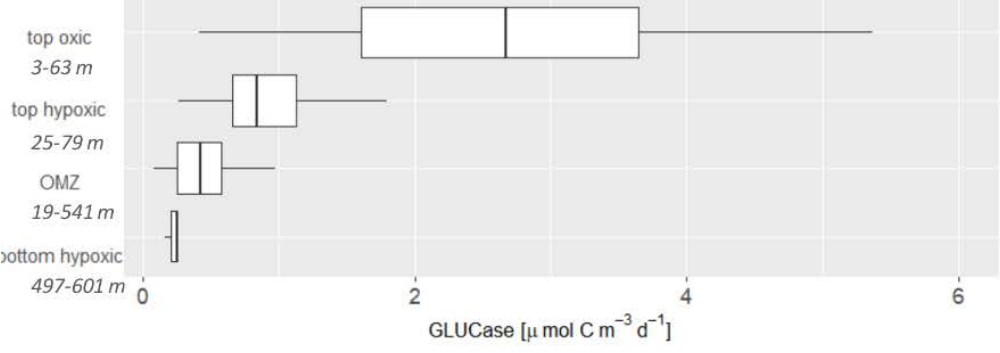

(c)

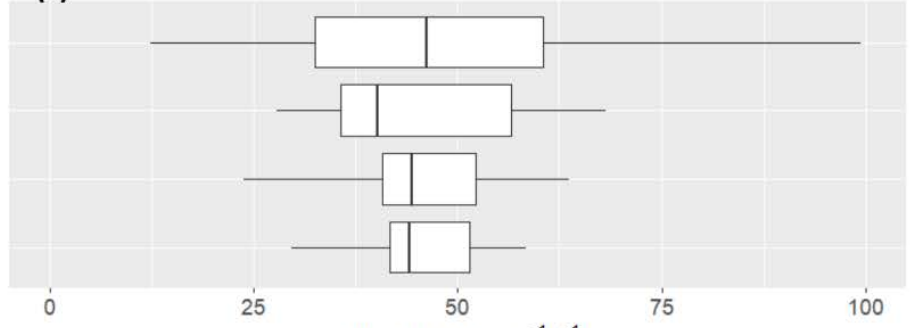

(d)

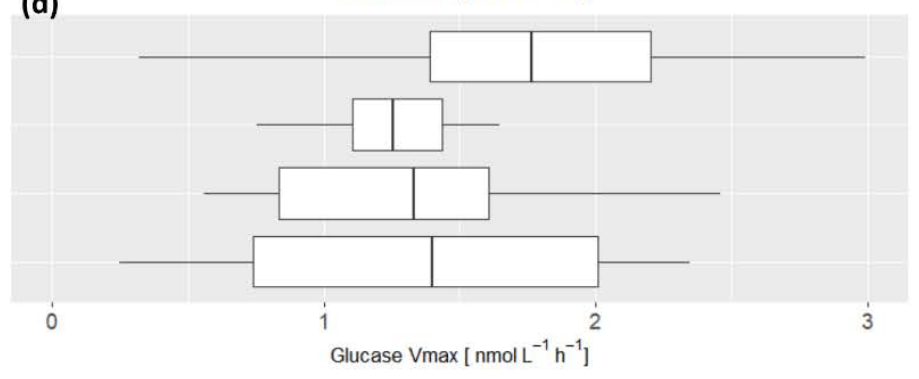

63

64

65 Supplementary Figure 1: Extracellular enzyme rates with all samples incubated under $\mathrm{N}_{2}$ atmosphere irrespective of the in situ oxygen concentration. Degradation rates of dissolved amino acids (DHAA) by leucine-aminopeptidase

67 (LAPase) (a), degradation rates of high molecular weight dissolved carbohydrates (DCHO) by ß-glucosidase

68 (GLUCase) (b) total potential LAPase rates $\left(\mathrm{V}_{\max }\right)(\mathbf{c})$, Glucase $\mathrm{V}_{\max }(\mathbf{d})$ at different oxygen regimes.

69

70

71

72

73

74

75

76 
81 Supplentary Table 1: Cruise, date, positions, sampled depths and bottom depth of all stations represented within the 82 manuscript. Extracellular enzyme rates were sampled at stations A-K, whereas some depths were not used for further 83 analyses, since the standard deviation was over 30\%. Bacterial biomass production was sampled at stations G-T. 84 Stations G-T were included in the estimation of carbon and oxygen loss rates. Cell abundance was sampled at every 85 station.

\begin{tabular}{|c|c|c|c|c|c|c|c|c|c|c|c|}
\hline \multirow{2}{*}{$\begin{array}{l}\text { Cruise } \\
\text { M138 }\end{array}$} & \multirow{2}{*}{$\begin{array}{l}\text { Station } \\
\text { A }\end{array}$} & \multirow{2}{*}{$\begin{array}{l}\text { Latitude } \\
-15.5393\end{array}$} & \multirow{2}{*}{$\begin{array}{l}\text { Longitude } \\
-75.6149\end{array}$} & \multirow{2}{*}{$\begin{array}{l}\text { Date } \\
06 / 19 / 2017\end{array}$} & \multicolumn{6}{|c|}{ Sampled Depth } & \multirow{2}{*}{$\begin{array}{l}\text { Bottom } \\
\text { Depth } \\
2507\end{array}$} \\
\hline & & & & & 3 & 17 & 24 & 28 & 39 & & \\
\hline & & & & & 99 & 197 & 396 & 596 & 1499 & & \\
\hline \multirow{2}{*}{ M138 } & B & -15.8595 & -76.1099 & $06 / 21 / 2017$ & 5 & 18 & 40 & 50 & 53 & & 2624 \\
\hline & & & & & 90 & 199 & 398 & 600 & & & \\
\hline \multirow[t]{2}{*}{ M138 } & C & -16.1593 & -76.5711 & $06 / 21 / 2017$ & 3 & 13 & 19 & 58 & 63 & & 3679 \\
\hline & & & & & 79 & 99 & 149 & 197 & 347 & 600 & \\
\hline \multirow[t]{2}{*}{ M138 } & D & -13.9971 & -76.6598 & $06 / 16 / 2017$ & 3 & 8 & 19 & 48 & 70 & & 586 \\
\hline & & & & & 98 & 117 & 197 & 397 & 498 & 541 & \\
\hline \multirow[t]{2}{*}{ M138 } & $\mathrm{E}$ & -14.2988 & -77.1796 & $06 / 17 / 2017$ & 19 & 38 & 47 & 66 & 97 & & 4702 \\
\hline & & & & & 147 & 198 & 299 & 398 & 497 & 601 & \\
\hline \multirow[t]{2}{*}{ M138 } & $\mathrm{F}$ & -14.7596 & -77.4829 & $06 / 23 / 2017$ & 9 & 28 & 48 & 53 & 99 & & 4154 \\
\hline & & & & & 148 & 197 & 297 & 397 & 497 & 601 & \\
\hline \multirow[t]{2}{*}{ M136 } & G & -12.2248 & -77.1795 & $04 / 27 / 2017$ & 4 & 20 & 25 & 30 & 39 & & 75 \\
\hline & & & & & 49 & 59 & 72 & & & & \\
\hline \multirow[t]{2}{*}{ M136 } & $\mathrm{H}$ & -12.3584 & -77.3621 & $04 / 25 / 2017$ & 3 & 6 & 27 & 39 & 48 & & 194 \\
\hline & & & & & 58 & 68 & 79 & 98 & 128 & & \\
\hline \multirow[t]{2}{*}{ M136 } & I & -12.453 & -77.4918 & $04 / 26 / 2017$ & 4 & 18 & 28 & 39 & 49 & & 403 \\
\hline & & & & & 59 & 73 & 199 & & & & \\
\hline \multirow[t]{2}{*}{ M136 } & J & -12.5807 & -77.6731 & $04 / 29 / 2017$ & 4 & 28 & 49 & 68 & 78 & & 973 \\
\hline & & & & & 99 & 195 & 393 & 448 & 498 & 598 & \\
\hline \multirow[t]{2}{*}{ M136 } & $\mathrm{K}$ & -12.338 & -78.0512 & $04 / 26 / 2017$ & 9 & 50 & 75 & 99 & 148 & & 1970 \\
\hline & & & & & 157 & 199 & 299 & 398 & 499 & 599 & \\
\hline \multirow[t]{2}{*}{ M136 } & $\mathrm{L}$ & -12.2782 & -77.2493 & $04 / 20 / 2017$ & 4 & 9 & 13 & 19 & 23 & & 130 \\
\hline & & & & & 28 & 43 & 48 & 58 & & & \\
\hline \multirow[t]{2}{*}{ M136 } & $M$ & -12.5806 & -77.6731 & 04/19/2017 & 3 & 19 & 39 & 55 & 65 & & 242 \\
\hline & & & & & 90 & 98 & 199 & 238 & & & \\
\hline \multirow[t]{2}{*}{ M136 } & $\mathrm{N}$ & -12.4134 & -77.4425 & 04/21/2017 & 3 & 18 & 50 & 99 & 124 & & 307 \\
\hline & & & & & 149 & 174 & 224 & 272 & 300 & & \\
\hline \multirow[t]{2}{*}{ M136 } & 0 & -12.5226 & -77.5834 & 04/20/2017 & 4 & 29 & 39 & 49 & 59 & & 751 \\
\hline & & & & & 79 & 89 & 98 & 147 & 597 & 745 & \\
\hline \multirow[t]{2}{*}{ M136 } & $P$ & -12.9873 & -78.2471 & $04 / 21 / 2017$ & 4 & 29 & 49 & 60 & 74 & & 5410 \\
\hline & & & & & 99 & 196 & 296 & 396 & 599 & 800 & \\
\hline \multirow[t]{2}{*}{ M136 } & $Q$ & -13.8938 & -76.5101 & 04.12 .2017 & 4 & 10 & 15 & 19 & 30 & & 166 \\
\hline & & & & & 39 & 50 & 69 & 99 & 128 & 159 & \\
\hline M136 & $\mathrm{R}$ & -14.1878 & -76.9312 & $04 / 13 / 2017$ & 5 & 14 & 29 & 49 & 81 & & 3042 \\
\hline & & & & & 101 & 123 & 199 & 399 & 599 & & \\
\hline M136 & $S$ & -14.3986 & -77.2389 & $04 / 14 / 2017$ & 1 & 49 & 89 & 99 & 148 & & 5149 \\
\hline & & & & & 198 & 299 & 399 & 499 & 599 & & \\
\hline M136 & $\begin{array}{ll}\mathrm{T} \\
\mathrm{T}\end{array}$ & -12.2254 & -77.1797 & $04 / 24 / 2017$ & 4 & 19 & 24 & 28 & 49 & & 76 \\
\hline
\end{tabular}

86

87

88 
89 Supplementary Table 2: Average and standard deviation at different oxygen regimes of all discussed parameters as

90 total rates (a) and cell-specific rates (b).

\begin{tabular}{|c|c|c|c|c|c|c|}
\hline \multicolumn{7}{|l|}{ a } \\
\hline parameter & station & oxygen regime & $\mathbf{n}$ & mean & SD & unit \\
\hline $\begin{array}{l}\text { cell } \\
\text { abundance }\end{array}$ & $A-T$ & $\begin{array}{l}\text { top oxic } \\
\text { top high hypoxic } \\
\text { top low hypoxic } \\
\text { OMz } \\
\text { bottom low hypoxic } \\
\text { top oxycline }\end{array}$ & $\begin{array}{l}52 \\
16 \\
20 \\
93 \\
14 \\
36 \\
\end{array}$ & $\begin{array}{l}19 \\
9 \\
8 \\
9 \\
1 \\
9\end{array}$ & $\begin{array}{l}9 \\
3 \\
3 \\
5 \\
0.1 \\
3 \\
\end{array}$ & cells $\times 10^{5} \mathrm{~mL}^{-1}$ \\
\hline $\begin{array}{l}\text { bacterial } \\
\text { production }\end{array}$ & G-T & $\begin{array}{l}\text { top oxic } \\
\text { top high hypoxic } \\
\text { top low hypoxic } \\
\text { OMz } \\
\text { bottom low hypoxic } \\
\text { oxycline }\end{array}$ & \begin{tabular}{|l}
34 \\
11 \\
17 \\
48 \\
5 \\
33 \\
\end{tabular} & $\begin{array}{l}194 \\
58 \\
43 \\
26 \\
5 \\
42 \\
\end{array}$ & $\begin{array}{l}189 \\
61 \\
38 \\
32 \\
3 \\
47 \\
\end{array}$ & $\mu \mathrm{mol} \mathrm{C} \mathrm{m}^{-3} \mathrm{~d}^{-1}$ \\
\hline \begin{tabular}{l}
\multicolumn{2}{l}{ degradation } \\
rates of \\
DHAA by \\
LAPase
\end{tabular} & $A-K$ & $\begin{array}{l}\text { top oxic } \\
\text { top high hypoxic } \\
\text { top low hypoxic } \\
\text { OMz } \\
\text { bottom low hypoxic }\end{array}$ & $\begin{array}{l}20 \\
6 \\
9 \\
41 \\
6 \\
\end{array}$ & $\begin{array}{l}13.9 \\
4.5 \\
2.4 \\
5.0 \\
2.4 \\
\end{array}$ & $\begin{array}{l}7.6 \\
3.0 \\
1.2 \\
1.8 \\
1.6 \\
\end{array}$ & $\mu \mathrm{mol} \mathrm{C} \mathrm{m}^{-3} \mathrm{~d}^{-1}$ \\
\hline \begin{tabular}{l}
\multicolumn{2}{l}{ degradation } \\
rates of \\
DCHO by \\
GLUCase
\end{tabular} & $A-K$ & $\begin{array}{l}\text { top oxic } \\
\text { top high hypoxic } \\
\text { top low hypoxic } \\
\text { OMz } \\
\text { bottom low hypoxic }\end{array}$ & $\begin{array}{l}22 \\
7 \\
8 \\
35 \\
3 \\
\end{array}$ & $\begin{array}{l}2.7 \\
1.5 \\
0.8 \\
0.7 \\
0.4\end{array}$ & $\begin{array}{l}2.5 \\
1.0 \\
0.5 \\
1.4 \\
0.3 \\
\end{array}$ & $\mu \mathrm{mol} C \mathrm{~m}-3 \mathrm{~d}-1$ \\
\hline $\begin{array}{l}\text { LAPase } \\
\text { Vmax }\end{array}$ & $A-K$ & $\begin{array}{l}\text { top oxic } \\
\text { top high hypoxic } \\
\text { top low hypoxic } \\
\text { OMz } \\
\text { bottom low hypoxic }\end{array}$ & $\begin{array}{l}22 \\
10 \\
10 \\
49 \\
6 \\
\end{array}$ & $\begin{array}{l}39.2 \\
41.1 \\
28.9 \\
49.9 \\
31.3 \\
\end{array}$ & \begin{tabular}{|l}
13.3 \\
24.0 \\
13.6 \\
22.1 \\
6.0 \\
\end{tabular} & $\mathrm{nmol} \mathrm{L} \mathrm{L}^{-1} \mathrm{~h}^{-1}$ \\
\hline $\begin{array}{l}\text { GLUCase } \\
\text { Vmax }\end{array}$ & $A-K$ & $\begin{array}{l}\text { top oxic } \\
\text { top high hypoxic } \\
\text { top low hypoxic } \\
\text { OMz } \\
\text { bottom low hypoxic }\end{array}$ & \begin{tabular}{|l}
26 \\
9 \\
11 \\
41 \\
4 \\
\end{tabular} & $\begin{array}{l}1.6 \\
1.4 \\
1.0 \\
1.6 \\
0.7\end{array}$ & $\begin{array}{l}0.6 \\
0.5 \\
0.3 \\
1.6 \\
0.4\end{array}$ & $\mathrm{nmol} \mathrm{L} \mathrm{L}^{-1} \mathrm{~h}^{-1}$ \\
\hline
\end{tabular}




\begin{tabular}{|c|c|c|c|c|c|c|}
\hline \multicolumn{7}{|l|}{ b } \\
\hline parameter & station & oxygen regime & $\mathbf{n}$ & mean & SD & unit \\
\hline $\begin{array}{l}\text { cell-specific } \\
\text { bacterial } \\
\text { productio }\end{array}$ & G-T & $\begin{array}{l}\text { top oxic } \\
\text { top high hypoxic } \\
\text { top low hypoxic } \\
\text { OMz } \\
\text { bottom low hypoxic } \\
\text { oxycline }\end{array}$ & $\begin{array}{l}34 \\
11 \\
17 \\
48 \\
5 \\
33\end{array}$ & $\begin{array}{l}92 \\
50 \\
46 \\
22 \\
35 \\
45\end{array}$ & $\begin{array}{l}78 \\
36 \\
27 \\
19 \\
23 \\
29\end{array}$ & amol $C$ cell d ${ }^{-1}$ \\
\hline \begin{tabular}{|lr|}
\multicolumn{2}{|l|}{ cell-specific } \\
Degradation \\
rates & of \\
DHAA & by \\
LAPase &
\end{tabular} & A-K & $\begin{array}{l}\text { top oxic } \\
\text { top high hypoxic } \\
\text { top low hypoxic } \\
\text { OMz } \\
\text { bottom low hypoxic }\end{array}$ & $\begin{array}{l}20 \\
6 \\
9 \\
41 \\
6\end{array}$ & $\begin{array}{l}6.5 \\
4.6 \\
3.1 \\
9.9 \\
21.6\end{array}$ & \begin{tabular}{|l|}
3.4 \\
3.1 \\
1.6 \\
6.8 \\
15.4 \\
\end{tabular} & amol $C$ cell d ${ }^{-1}$ \\
\hline \begin{tabular}{|lr|}
\multicolumn{2}{|l|}{ cell-specific } \\
Degradation \\
rates & of \\
DCHO & by \\
GLUCase & \\
\end{tabular} & A-K & $\begin{array}{l}\text { top oxic } \\
\text { top high hypoxic } \\
\text { top low hypoxic } \\
\text { OMz } \\
\text { bottom low hypoxic }\end{array}$ & $\begin{array}{l}22 \\
7 \\
8 \\
35 \\
3\end{array}$ & $\begin{array}{l}1.3 \\
1.4 \\
1.1 \\
1.1 \\
3.2\end{array}$ & \begin{tabular}{|l|}
1.6 \\
1.1 \\
0.8 \\
1.6 \\
3.2 \\
\end{tabular} & amol $C$ cell d ${ }^{-1}$ \\
\hline $\begin{array}{l}\text { cell-specific } \\
\text { LAPase } \\
\text { Vmax }\end{array}$ & $A-K$ & $\begin{array}{l}\text { top oxic } \\
\text { top high hypoxic } \\
\text { top low hypoxic } \\
\text { OMz } \\
\text { bottom low hypoxic }\end{array}$ & $\begin{array}{l}22 \\
10 \\
10 \\
49 \\
6\end{array}$ & $\begin{array}{l}23.1 \\
44.1 \\
39.9 \\
103.5 \\
274.1 \\
\end{array}$ & \begin{tabular}{|l|}
15.1 \\
29.7 \\
23.0 \\
114.2 \\
51.7 \\
\end{tabular} & amol cell ${ }^{-1} h^{-1}$ \\
\hline $\begin{array}{l}\text { cells-specific } \\
\text { GLUCase } \\
\text { Vmax }\end{array}$ & A-K & $\begin{array}{l}\text { top oxic } \\
\text { top high hypoxic } \\
\text { top low hypoxic } \\
\text { OMz } \\
\text { bottom low hypoxic }\end{array}$ & $\begin{array}{l}26 \\
9 \\
11 \\
41 \\
4\end{array}$ & $\begin{array}{l}0.9 \\
1.5 \\
1.3 \\
2.7 \\
6.0\end{array}$ & $\begin{array}{l}0.4 \\
0.6 \\
0.5 \\
2.4 \\
3.6\end{array}$ & amol cell ${ }^{-1} \mathrm{~h}^{-1}$ \\
\hline
\end{tabular}


110 Both, G. W., McInnes, J. L., Hanlon, J. E., May, B. K. and Elliott, W. H.: Evidence for an accumulation of 111 messenger RNA specific for extracellular protease and its relevance to the mechanism of enzyme secretion in 112 bacteria, J. Mol. Biol., 67(2), 199-217, doi:10.1016/0022-2836(72)90236-7, 1972.

113 Elstner, E. F., Ed.: Der Sauerstoff: Biochemie, Biologie, Medizin, BI-Wiss_Verl, Mannheim., 1990.

114 Endres, S., Galgani, L., Riebesell, U., Schulz, K.-G. and Engel, A.: Stimulated Bacterial Growth under Elevated 115 pCO2: Results from an Off-Shore Mesocosm Study, edited by S. Dupont, PLoS One, 9(6), e99228, 116 doi:10.1371/journal.pone.0099228, 2014.

117 Fischer, T., Banyte, D., Brandt, P., Dengler, M., Krahmann, G., Tanhua, T. and Visbeck, M.: Diapycnal oxygen 118 supply to the tropical North Atlantic oxygen minimum zone, Biogeosciences, 10(7), 5079-5093, doi:10.5194/bg-10119 5079-2013, 2013.

120 Osborn, T. R.: Estimates of the local rate of vertical diffusion from dissipation measurements, J. Phys. Oceanogr., 121 10(1), 83-89, doi:10.1175/1520-0485(1980)010<0083:EOTLRO>2.0.CO;2, 1980.

122 Piontek, J., Borchard, C., Sperling, M., Schulz, K. G., Riebesell, U. and Engel, A.: Response of bacterioplankton 123 activity in an Arctic fjord system to elevated $\mathrm{pCO}_{2}$ : results from a mesocosm perturbation study, Biogeosciences, 124 10(1), 297-314, doi:10.5194/bg-10-297-2013, 2013.

125 Schafstall, J., Dengler, M., Brandt, P. and Bange, H.: Tidal-induced mixing and diapycnal nutrient fluxes in the 126 Mauritanian upwelling region, J. Geophys. Res., 115(C10), C10014, doi:10.1029/2009JC005940, 2010. 